\title{
Early diagnosis and adulthood transition in autism spectrum disorders (ASD): How primary care helps to close the gaps
}

\author{
Jun Liu ${ }^{1}$, Morgane Amat ${ }^{2}$, Rui Song ${ }^{3}$ and Xuejun Kong1,3* \\ ${ }^{1}$ Massachusetts General Hospital, Harvard Medical School, USA \\ ${ }^{2}$ Harvard School of Dental Medicine, USA \\ ${ }^{3}$ To Cure Autism institute, American Chinese Medical Exchange Society, USA
}

\begin{abstract}
Autism spectrum disorder (ASD), a complex neurodevelopmental condition, is a rapidly expanding public health crisis worldwide. The current care system for ASD is fragmented and inadequate. The medical community recognizes two major gaps as deserving of immediate attention: early screening and the transition to adulthood. In this essay, we emphasize the critical status of these two missing components and their negative impacts on ASD care. We also explore the systemic causes of these unmet needs, discuss how a primary care-based approach is best suited to close the gaps, and briefly introduce some innovative primary care-based interventions currently under way. Finally, we identify obstacles and propose steps to overcome these barriers in order to achieve timely diagnosis and continuity of care for patients with ASD.
\end{abstract}

\section{Introduction: The ASD "Epidemic": Identify major gaps in management}

Autism Spectrum Disorder (ASD) is a fast-growing neurodevelopmental disorder characterized by deficits in social communication, as well as restricted or repetitive behaviours and interest [1,2]. Its global prevalence has reached around 1 in 160 [3]. In the United States (U.S.), prevalence is 1 in 68 and continues to rise [4]. Despite significant global efforts to improve healthcare for patients with ASD, this population is still largely underserved [5-7]. In particular, early screening and adult transition services are two major unmet needs [8-10]. The most vulnerable and underserved sub- populations in the current landscape of ASD care include highrisk infants (e.g., siblings of patients with ASD or those with certain prenatal exposures), undiagnosed patients with ASD, and adolescents or young adults with ASD [11]. Recently, leading ASD tertiary-care centers in the U.S., including Boston Children's Hospital, have begun to address these challenges [12].

\section{Early diagnosis and adulthood transition: The unmet needs}

Early screening and timely diagnosis of populations at high risk for ASD is currently inadequate. Studies have shown that early detection of ASD, especially before age 2, followed by early intervention, can lead to better outcomes $[13,14]$. Clinicians can reliably diagnose ASD in children as young as 24 months, yet a recent systematic review demonstrated that the mean age at diagnosis ranges from 38 to 120 months [15]. A key factor in this delay in diagnosis is the lack of endorsement by the US Preventive Services Task Force (USPSTF), which concluded that there is insufficient evidence to support universal ASD screening. This, together with other factors, led to the poor implementation rate of universal, tier-1 screening instruments such as M-CHAT (Modified Checklist for Autism in Toddlers) starting at 18 months [15-18]. In fact, only approximately $50 \%$ of all US paediatric practices adopted routine universal screening for ASD [19]. The stance of USPSTF is controversial; many experts in the field and professional organizations such as the American Academy of Paediatrics believe that strong evidence already exist to support universal screening [20].

Several groups are currently validating tier-2 screening for children at risk [21]. In fact, some paediatric practices already adopted tier-2 screening instruments and recently reported reductions in diagnostic delays via partnership with specialists [22]. However, it is currently largely absent from standard paediatric practices. Furthermore, a growing body of evidence suggests that high-risk infants and toddlers warrant earlier screening or specialist referrals before 18 months [23]. Through observation, parents and clinicians have noted early signs of ASD as well as objective measurements at or before 12 months of age $[21,24]$. While studies confirmed that paediatricians fail to refer a high proportion of at-risk children for timely evaluation [8,21,22,25,26], some early screening tools have already shown promising sensitivities and specificities for early detection [21]. A shortage of ASD specialists, as well as poor coordination between primary care providers (PCPs) and specialty care, can further delay confirmatory diagnosis and treatment, contributing to suboptimal outcomes [27].

In addition to early detection, paediatric-to-adult transition is another important unmet need [10]. Approximately 50,000 Americans with ASD turn 18 years old each year, requiring structured transition

${ }^{\star}$ Correspondence to: Xue-Jun Kong, Synapse Program, A. A. Martinos Center for Biomedical Imaging, Massachusetts General Hospital, Harvard Medical School, Charlestown, MA, USA, Tel: 617-726-9439; E-mail: Xkong1@mgh.harvard.edu

Key words: autism spectrum disorder, autism, healthcare, physicians, primary care, early diagnosis, transition to adult care, dental care, tertiary care hospital, neurodevelopmental disorders

Received: September 11, 2018; Accepted: September 24, 2018; Published: September 28, 2018 
services with unique considerations from both the medical and social perspective [28].

Unfortunately, the current U.S. healthcare system has few structured medical transition protocols in place $[29,30]$. Fewer than $20 \%$ of young people with ASD receive healthcare transition services, and fewer than $50 \%$ have the opportunity to discuss the transition to an adult provider with their paediatricians [31]. Paediatricians lack transition-related training [32], while Internists report insufficient knowledge in ASD care [33]. Consequently, most patients with ASD continue in paediatric care beyond the age of twenty-two [34]. Unstructured transition may lead to a loss to care [35] which has been associated with increases in emergency room attendance, suicides, homicides, and psychotic breakdowns in this age group [10,35,36]. This period also coincides with an increase in obesity, epilepsy, depression, and anxiety $[35,37,38]$. The increased morbidity and loss of care has formed a consensus among the medical community that adolescents with ASD are vulnerable and underserved, requiring our immediate attention [10].

\section{“Resuscitating" PCPs' roles in ASD care}

Traditionally, psychiatry, developmental paediatrics, and neurology form the cornerstone of ASD management in the U.S. [39] and most other countries [40-42], while PCPs play a very limited role in ASD care $[3,4,33,43]$. It's notable that PCPs make up nearly $50 \%$ of the total physician population in the U.S. [44,45] and in most other developed countries [46-49]. PCPs currently perform well visits, making them well suited for early screening and providing resources for transition to adulthood [50,51]. In contrast, specialists in ASD care are becoming increasingly scarce. Furthermore, their expertise is best utilized for confirmative diagnosis and specific treatments rather than screening and health maintenance. With the fast-growing population of ASD patients and at-risk infants, PCP's roles in ASD routine care and co-management warrant prompt "resuscitation", especially in the areas of early detection and adult transition.

In recent years, the U.S. has seen an expansion of ASD centers affiliated with tertiary-care hospitals. However, none of these have inhouse primary-care services, and there is limited collaboration between ASD centers and primary-care practices in the community [53]. Notably, several primary care-oriented efforts to improve care for patients with ASD have been successfully piloted $[8,9]$, and neuropsychiatry-based ASD centers are working to build stronger alliances with primary-care practices in the community [52]. A primary-care based approach, or "activated autism practice," in the words of Kairys et al. [9] could serve as an example.

\section{How PCPs help to close the gaps in early diagnosis}

To improve early diagnosis of ASD, paediatric practices need to adopt evidence-based protocols necessary to provide timely screening during well-child visits. The training goals should be two- fold: to implement universal screening with validated tools [51], and to train paediatricians to recognize high-risk patients, so that these toddlers (and even infants) can be identified and referred for timely evaluation. A study by Kairys et al. [9] illustrates the success of such an educational intervention among PCPs.

A recent systematic review of tier-2 screening research concluded insufficient evidence due to small number of studies related to each published screening tool [21]. However, attempts to validate existing tier-2 screening tools are currently under way $[21,54,55]$. Similarly, research efforts are growing for the development of effective tools for early detection $[21,56]$. These new approaches may allow for ASD diagnosis and intervention as early as $12-15$ months of age $[21,24,56]$. New technologies, such as eye-tracking devices, could be integrated into primary-care practice for early screening of children at high risk of developing ASD [57]. We believe that in the near future, there will be more convincing evidence to recommend early screening for high risk infants/toddlers. Leaders of paediatric practices need to stay up-todate with the fast-evolving landscape of ASD screening. However, as current early screening tools are undergoing validation, we believe that it is important for paediatricians to proactively refer at-risk infants/ toddlers to early intervention services and/or specialists for timely evaluation. Figure 1 presents our proposal for a PCP-led ASD early detection program with consideration of tier-2 screening.

To further expedite diagnosis and management of comorbidities, PCPs can partner with a pre- selected network of developmental paediatricians, psychiatrists, and early intervention clinicians for fast-track diagnosis and treatment. ASD screening of the paediatric population at large will nonetheless be time consuming. PCP offices with trained staff and clear protocols are essential to ensure the success and reliability of early screening.

\section{PCP-led transition team smooths transition into adult care}

PCPs, with their large physician base and focus on health maintenance, are ideally positioned to take the lead in coordinating the creation and implementation of transition services. Primary-care practices, with guidance from specialists, can improve transition care via a three-pronged approach. First, they should set up a dedicated transition team bringing together frontline paediatricians and internists assisted by case managers, behavioural therapists, mental health providers, and social workers. Second, the team should design working protocols that clearly delineate workflow and duties for each team member, with both outpatient and inpatient navigation algorithms. Third, all team members and related staff should undergo transition-related training. Internists are often less familiar with ASD care than paediatricians [44] and may require additional ASD-specific training to successfully implement transition protocols.

The creation and implementation of transition protocols should be guided by the following goals. First, they serve to address teen or young adults with ASD's medical and mental health concerns as well as provide sex education in a stepwise, evidence-based, and ASDfriendly manner. The secondary goals are to coordinate with schools, employment training programs, and other community resources to prepare patients for greater functional independence in society. With a dedicated team, systematic protocols, and physician training, the PCP-led transition services we propose (Figure 2) may help to minimize missed days from school and work, emergency room visits, hospitalizations, suicides, and crime rates within the population of adolescents with ASD. U.S. primary-care practices affiliated with major tertiary-care ASD treatment centers, such as that of Boston Children's Hospital, have already begun to address the urgent issues of ASD care transition, although details are still unclear [12].

\section{Challenges and future perspectives}

ASD is a complex neurodevelopmental disorder with high prevalence [1,3]. Two major gaps in the current landscape of ASD management are early diagnosis and paediatric-to-adult transition. Based on what we have learned from the experiences of patients, families, and providers, we believe that a primary care-based approach 


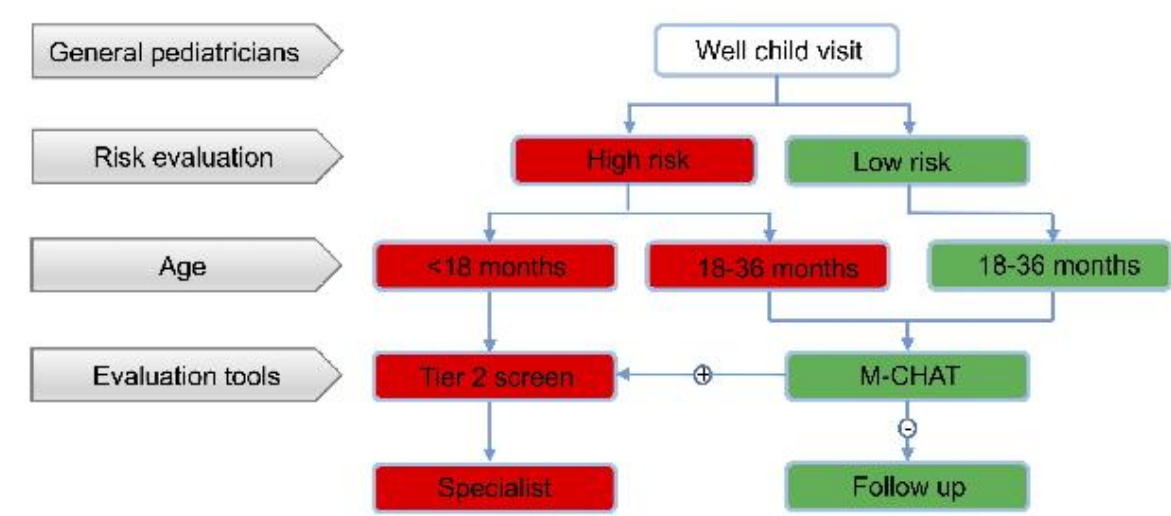

Figure 1. PCP-led early-screening protocol. Early screening teams led by primary care physicians can perform evidence-based universal screening and tier-2 screening to facilitate early diagnosis and intervention for patients with ASD

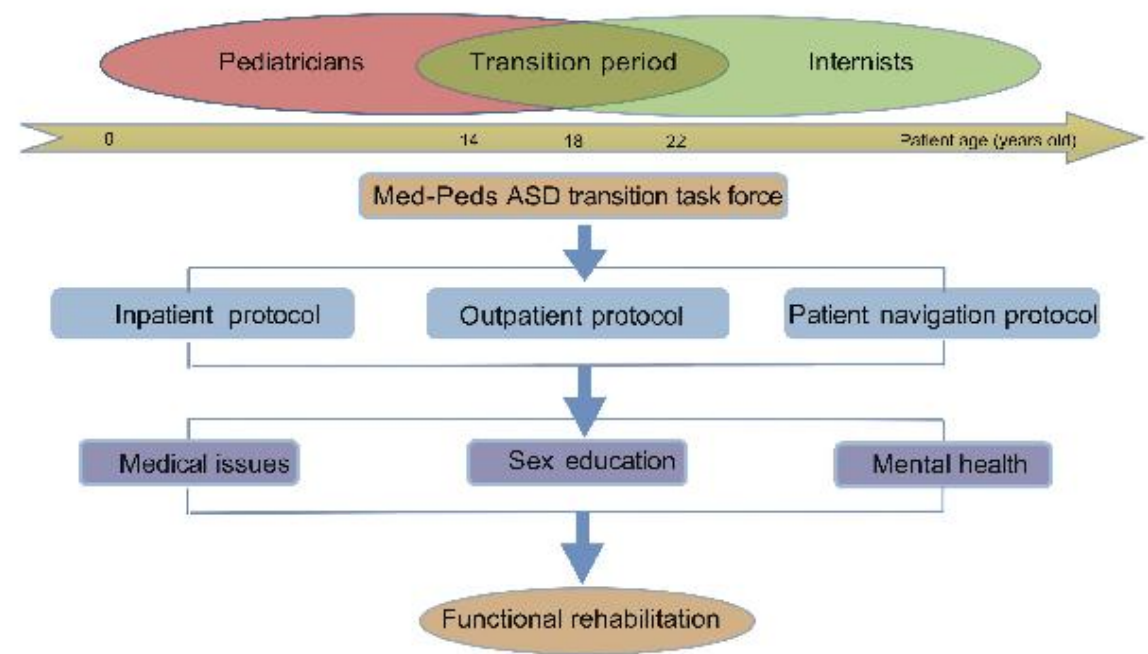

Figure 2. PCP-led adult transition program. During the transition period of patients with ASD (14 to 22 years of age), paediatricians and internists in primary care practices can come together to develop and implement transition protocols (including inpatient, outpatient, and patient navigation components). These protocols should aim to address patients' medical and mental health concerns and improve sex education which tends to be overlooked for adolescents and young adults with ASD

may be the key to fulfilling future needs. However, we must first overcome significant obstacles. Successful early screening requires upto-date paediatrician training, team building, protocol implementation, as well as parental awareness. The same applies to the transition to adult care, with the additional need for ASD-specific training of internists.

Recently, the Center for Disease Control implemented an ASD awareness campaign, reflecting increased governmental support for the issues identified above [58]. The American Association of Paediatrics support the notion of early screening. However, improved ASD care will require changes in insurance reimbursement, policy support such as recommendation from the Task force, and dedicated funding. Ongoing research efforts serve to delineate how PCPs can engage in further co-management roles alongside specialists to fill the current gaps in the management of ASD [59].

Primary care systems are already in place or expanding in most western countries with a high prevalence of ASD [46,47,49]. We call for the incorporation of primary care within existing ASD centers, and better collaboration between ASD centers and PCPs in the community. By promoting early diagnosis and improving transition to adulthood, PCPs worldwide can help individuals with ASD reach their maximal functional status, which in turn will benefit the society as a whole.

\section{Compliance with ethical standards}

Funding: This study was funded by the MGH fund 230361.

Ethical approval: This article does not contain any studies with human participants or animals performed by any of the authors.

\section{References}

1. Elsabbagh M, Divan G, Koh Y-J, Kim YS, Kauchali S, Marcín C (2012) Global Prevalence of Autism and Other Pervasive Developmental Disorders. Autism Res 5: 160-179. [Crossref]

2. Neggers YH (2014) Increasing Prevalence, Changes in Diagnostic Criteria, and Nutritional Risk Factors for Autism Spectrum Disorders. ISRN Nutrition 2014: $1-14$.

3. WHO (2017) Autism spectrum disorders fact sheet. World Health Organization, Media Centre. Available at: http://www.who.int/mediacentre/factsheets/autism-spectrumdisorders/en/.

4. Christensen DL, Baio J, Van Naarden Braun K, Bilder D, Charles J, et al. (2016) Prevalence and Characteristics of Autism Spectrum Disorder Among Children Aged 8 Years - Autism and Developmental Disabilities Monitoring Network, 11 Sites, United States, 2012. MMWR Surveill Summ 65: 1-23. [Crossref]

5. Kornblau BL (2014) The Case for Designating People with Intellectual and Developmental Disabilities as a Medically Underserved Population. Autistic Self Advocacy Network (ASAN), Policy Brief. 
6. Sobotka SA, Francis A, Vander, Ploeg, Booth K (2016) Associations of family characteristics with perceptions of care among parents of children with autism. Child Care Health Dev 42: 135-140. [Crossref]

7. Zablotsky B, Kalb LG, Freedman B, Vasa R, Stuart EA (2014) Health Care Experiences and Perceived Financial Impact Among Families of Children with an Autism Spectrum Disorder. Psychiatr Serv 65: 395-398. [Crossref]

8. Carbone PS, Norlin C, Young PC (2016) Improving Early Identification and Ongoing Care of Children with Autism Spectrum Disorder. Pediatrics 137: e1-e9.

9. Kairys SW, Petrova A (2016) Role of Participation of Pediatricians in the "Activated Autism Practice" Program in Practicing Children with Autism Spectrum Disorders at the Primary Care Setting. Glob Pediatr Health 3: 4-6. [Crossref]

10. Murphy CM, Wilson CE, Robertson DM, Ecker C, Daly EM, Hammond N (2016) Autism spectrum disorder in adults: diagnosis, management, and health services development. Neuropsychiatr Dis Treat 12:1669-1686. [Crossref]

11. Karimi P, Kamali E, Mousavi SM, Karahmadi M (2017) Environmental factors influencing the risk of autism. J Res Med Sci 22: 27. [Crossref]

12. Transitioning to adult care. Boston Children's Primary Care at Longwood. Available at: http://www.childrenshospital.org/centers-and-services/programs/a-_-e/childrenshospital- primary-care-center-chpcc-program/transitioning-to-adult-care.

13. Daniels AM, Mandell DS (2014) Explaining differences in age at autism spectrum disorder diagnosis: A critical review. Autism 18: 583-597. [Crossref]

14. Elder J, Kreider C, Brasher S, Ansell M (2017) Clinical impact of early diagnosis of autism on the prognosis and parent-child relationships. Psychol Res Behav Manag 10: 283-292. [Crossref]

15. Barton ML, Dumont-Mathieu T, Fein D (2012) Screening young children for autism spectrum disorders in primary practice. J Autism Dev Disord 42: 1165-1174. [Crossref]

16. Siu AL, Bibbins-Domingo K, Grossman DC, Baumann LC, Davidson KW, et al (2016) Screening for Autism Spectrum Disorder in Young Children: US Preventive Services Task Force Recommendation Statement. JAMA 315; 691-696 [Crossref]

17. Gordon-Lipkin E, Foster J, Peacock G (2016) Whittling Down the Wait Time: Exploring Models to Minimize the Delay from Initial Concern to Diagnosis and Treatment of Autism Spectrum Disorder. Pediatr Clin North Am 63: 851-859.

18. Janvier YM, Harris JF, Coffield CN, Louis B, Xie M, et al. (2016) Screening for autism spectrum disorder in underserved communities: Early childcare providers as reporters. Autism 20: 364-373.

19. Pierce K, Courchesne E, Bacon E (2016) To Screen or Not to Screen Universally for Autism is not the Question: Why the Task Force Got It Wrong. J Pediatr 176: 182-194. [Crossref]

20. American Academy of Pediatrics. AAP Statement on U.S. Preventive Services Task Force Final Recommendations on Autism Screening. Feb 2016. Available at: https:// www.aap.org/en- us/about-the-aap/Committees-Councils-Sections/Council-onChildren-with- Disabilities/Pages/Recent-Information.aspx.

21. Towle PO, Patrick PA (2016) Autism Spectrum Disorder Screening Instruments for Very Young Children: A Systematic Review. Autism Res Treat 02: 1-29.

22. Lemay JF, Yohemas M, Langenberger S (2018) Redesign of the autism spectrum screening and diagnostic process for children aged 12 to 36 months. Paediatr Child Health 23: 308-313. [Crossref]

23. Rotholz DA, Kinsman AM, Lacy KK, Charles J (2017) Improving Early Identification and Intervention for Children at Risk for Autism Spectrum Disorder. Pediatrics 139: 1-7.

24. Olliac B, Crespin G, Laznik M-C, Cherif Idrissi El, Ganouni O, et al. (2017) Infant and dyadic assessment in early community-based screening for autism spectrum disorder with the PREAUT grid. Botbol M (ed.) PLoS ONE 12: e0188831-22.

25. Morelli DL, Pati S, Butler A, Blum NJ, Gerdes M, et al. (2014) Challenges to implementation of developmental screening in urban primary care: a mixed methods study. BMC Pediatr 14: 16. [Crossref]

26. Windham GC, Smith KS, Rosen N, Anderson MC, Grether JK, et al. (2014) Autism and Developmental Screening in a Public, Primary Care Setting Primarily Serving Hispanics: Challenges and Results. J Autism Dev Disord 44: 1621-1632. [Crossref]

27. Daniels AM, Halladay AK, Shih A, Elder LM, Dawson G (2014) Approaches to enhancing the early detection of autism spectrum disorders: a systematic review of the literature. J Am Acad Child Adolesc Psychiatry 53: 141-152. [Crossref]

28. Roux AM, Shattuck PT, Cooper BP, Anderson KA, Wagner M, et al. (2013) Postsecondary Employment Experiences Among Young Adults with an Autism Spectrum Disorder. J Am Acad Child Adolesc Psychiatry 52: 931-939. [Crossref]
29. Hatfield M, Falkmer M, Falkmer T, Ciccarelli M (2016) Evaluation of the effectiveness of an online transition planning program for adolescents on the autism spectrum: trial protocol. Child Adolesc Psychiatry Ment Health 10: 48. [Crossref]

30. Sarris M (2015) Interactive Autism Network at Kennedy Krieger Institute. Leaving the pediatrician: charting the medical transition of youth with autism. Sept 21. Available at: https://iancommunity.org/ssc/medical- transition-youth-autism.

31. Cheak-Zamora NC, Farmer JE, Mayfield WA, Clark MJ, Marvin AR, et al. (2014) Health care transition services for youth with autism spectrum disorders. Rehabil Psychol 59: 340-348. [Crossref]

32. Kuhlthau KA, Warfield ME, Hurson J, Delahaye J, Crossman MK (2015) Pediatric provider's perspectives on the transition to adult health care for youth with autism spectrum disorder: Current strategies and promising new directions. Autism 19: 262271. [Crossref]

33. Bruder MB, Kerins G, Mazzarella C, Sims J, Stein N (2012) Brief report: the medical care of adults with autism spectrum disorders: identifying the needs. J Autism Dev Disord 42: 2498-2504. [Crossref]

34. Rogers K, Zeni MB (2015) Systematic review of medical home models to promote transitions to primary adult health care for adolescents living with autism spectrum disorder. Worldviews Evid Based Nurs 12: 98-107. [Crossref]

35. Volkmar FR, Jackson, SLJ, Hart L (2017) Transition Issues and Challenges for Youth with Autism Spectrum Disorders. Pediatric Annals 46: e219-223. [Crossref]

36. Chandrasekhar T, Sikich L (2015) Challenges in the diagnosis and treatment of depression in autism spectrum disorders across the lifespan. Dialogues Clin Neurosci 17: 219-227. [Crossref]

37. van Schalkwyk GI, Volkmar FR (2017) Autism Spectrum Disorders: Challenges and Opportunities for Transition to Adulthood. Child Adolesc Psychiatr Clin N Am 26: 329-339. [Crossref]

38. Vasa RA, Mazurek MO (2015) An update on anxiety in youth with autism spectrum disorders. Curr Opin Psychiatry 28: 83-90. [Crossref]

39. Ming X, Hashim A, Fleishman S, West T, Kang N, et al. (2011) Access to specialty care in autism spectrum disorders-a pilot study of referral source. BMC Health Serv Res 11: 99. [Crossref]

40. Buescher AV, Cidav Z, Knapp M, Mandell DS (2014) Costs of autism spectrum disorder in the United Kingdom and the United States. JAMA Pediatr 168: 721-728. [Crossref]

41. Ouellette-Kuntz H, Coo H, Lam M, Breitenbach MM, Hennessey PE, et al. (2014) The changing prevalence of autism in three regions of Canada. J Autism Dev Disord 44 120-136. [Crossref]

42. Pantelis PC, Kennedy DP (2016) Estimation of the prevalence of autism spectrum disorder in South Korea, revisited. Autism 20: 517-527. [Crossref]

43. Luiselli JK (2015) Review of Behavioral Training and Performance Management Interventions with Autism Spectrum Disorder (ASD) Care-Providers. Int J Behav Anal Aut Spect Dis 1: 9-17.

44. Hing E, Hsiao CJ (2014) State Variability in Supply of Office-based Primary Care Providers: United States, 2012. Centers for Disease Control and Prevention, National Center for Health Statistics, Data Brief No. 151.

45. HIS Inc (2018) The Complexities of Physician Supply and Demand: Projections from 2013 to 2025 Final Report. Association of American Medical Colleges. 2015 Mar. Available at: https://www.aamc.org/download/426242/data/ihsreportdownload. pdf?cm_mmc=AAMC_-_ ScientificAffairs-_PDF__-ihsreport.

46. Djalali S, Meier T, Hasler S, Rosemann T, Tandjung R (2015) Primary care in Switzerland gains strength. Fam Pract 32: 348-353. [Crossref]

47. Hutchison B, Levesque JF, Strumpf E, Coyle N (2011) Primary health care in Canada: systems in motion. Milbank $Q$ 89: 256-288. [Crossref]

48. Porter ME, Guth C (2012) The German Health Care System: Overview and Historica Development. In: Redefining German Health Care. Redefining German Health Care 12: 53-69.

49. Roland M, Guthrie B, Thomé DC (2012) Primary medical care in the United Kingdom. J Am Board Fam Med 25 Suppl 1: S6-11. [Crossref]

50. Cox JV, Kirschner N (2008) Patient-centered medical home: renewing primary care. $J$ Oncol Pract 4: 285-286. [Crossref]

51. Nicolaidis C, Kripke CC, Raymaker D (2014) Primary Care for Adults on the Autism Spectrum. Med Clin North Am 98: 1169-1191. [Crossref]

52. Brookman-Frazee L, Stahmer AC, Lewis K, Feder JD, Reed S (2012) Building A Research-Community Collaborative to Improve Community Care for Infants and Toddlers At-Risk for Autism Spectrum Disorders. J Community Psychol 40: 715-734. [Crossref] 
53. Toh, T-H, Tan, VW-Y, Lau, PS-T, Kiyu A (2017) Accuracy of Modified Checklist for Autism in Toddlers (M-CHAT) in Detecting Autism and Other Developmental Disorders in Community Clinics. J Autism Dev Disord 118: 405-408.

54. Choueiri R, Wagner S (2015) A New Interactive Screening Test for Autism Spectrum Disorders in Toddlers. J Pediatr 167: 460-466. [Crossref]

55. Zwaigenbaum L, Bauman ML, Choueiri R, Kasari C, Carter A, et al. (2015) Early Intervention for Children with Autism Spectrum Disorder Under 3 Years of Age: Recommendations for Practice and Research. Pediatrics 136: 60-S81. [Crossref]

56. Sacrey L-AR, Zwaigenbaum L, Bryson S, Brian J, Smith IM, et al. (2018) Parent and clinician agreement regarding early behavioral signs in 12- and 18-month-old infants at-risk of autism spectrum disorder. Autism Res 101: 213-219.
57. Altman S, O'Connor S, Anapolsky E, Sexton L (2014) Federal and state benefits for transition age youth. J Pediatr Rehabil Med 7: 71-77. [Crossref]

58. Act Early Ambassadors. Centers for Disease Control and Prevention, Learn the Signs. Available at: https://www.cdc.gov/ncbddd/actearly/ambassadors-list.html.

59. Autism Speaks Autism Treatment Network. Research Opportunity - Expanding Primary Care for Children with ASD. Available at: http://asatn.org/node/873.

60. Fang J, Egorova N, Rong P, Liu J, Hong Y, et al. (2016) Early cortical biomarkers of longitudinal transcutaneous vagus nerve stimulation treatment success in depression. Neuroimage Clin 14: 105-111. [Crossref]

61. Merritt, Hawkins (2017) Physician Supply Considerations: The Emerging Shortage of Medical Specialists. White Paper Series. 1-16.

Copyright: $(2018$ Liu J. This is an open-access article distributed under the terms of the Creative Commons Attribution License, which permits unrestricted use, distribution, and reproduction in any medium, provided the original author and source are credited. 